\title{
Rauchen als Risikofaktor in der Traumatologie
}

\author{
Weltweit rauchen über 1,3 Billionen Menschen, etwa 6 Millio- \\ nen Menschen sterben jährlich an den Folgen des Zigaretten- \\ konsums. Die Wirkung auf die Organsysteme wurde intensiv \\ erforscht. Der Effekt auf das muskulo-skelettale System wird \\ jedoch oft unterschätzt. Diese Studie untersucht die Fraktur- \\ heilung, Weichteilverhältnisse und die Zeit bis zur knöchernen \\ Konsolidierung bei Patienten mit Tabak-Konsum. \\ Scolaro, JA et al. Cigarette Smoking Increases Complications Following Fracture. A Sys- \\ tematic Review J Bone Joint Surg Am. 2014; 96: 674-681
}

\section{Material und Methoden $\nabla$}

Die größten medizinischen Datenbanken wurden systematisch mit verschiedenen Schlüsselwörtern durchsucht. Die relevanten Daten wurden selektiert und mit Daten von Nicht-Rauchern verglichen.

\section{Ergebnisse \\ $\nabla$}

Von initial 7110 Fachartikeln konnten letztlich 19 Artikel ( 7 prospektive Studien, 12 retrospektive Studien) in diese Analyse eingeschlossen werden. Die Studien umfassten insgesamt 6374 Frakturen (6356 Patienten) von 1446 Rauchern und 4910 Nicht-Rauchern. Die offenen oder ge- schlossenen Frakturen betrafen Tibia, Femur, Humerus, distale Fibula und multiple Röhrenknochen. Die Therapie erfolgte konservativ oder operativ.

Die Nonunion-Rate war bei Rauchern mit frischen Frakturen der langen Röhrenknochen signifikant höher (12\%), bei offenen Frakturen um 9\% höher. Die Zeit bis zur knöchernen Konsolidierung war mit durchschnittlich 30 Wochen bei geschlossenen Frakturen ebenfalls höher gegenüber 24 Wochen bei Nichtrauchern, jedoch nicht signifikant. Die Konsolidierungszeit offener Frakturen lag bei Rauchern bei im Schnitt 37 Wochen gegenüber 29 Wochen bei Nichtrauchern. Die Rate von oberflächlichen und tiefen
Weichteilinfektionen war jeweils ebenfalls höher bei Zigarettenkonsum, aber nicht signifikant.

\section{Kommentar}

Die vorliegende Studie zeigt eindrücklich, dass Nikotinkonsum auch in der Traumatologie einen wichtigen, die Heilung beeinflussenden Faktor, darstellt. Die deutlich erhöhte Rate verzögerter knöcherner Konsolidierungszeit sowie das Auftreten von Nonunion beinhalten als Folge natürlich auch eine (hier nicht betrachtete) Erhöhung von Re-Operationen, Folgeschäden und verlängerter Arbeitsunfähigkeit.

Daher sollten die Patienten sowohl präals auch postoperativ ausführlich über die Risiken des Nikotinkonsums hinsichtlich der Heilungsgefährdung aufgeklärt werden. Die hier festgestellten Daten lassen sich vermutlich auch auf die Heilung bei Arthrodesen, Osteitiden und Umstellungsosteotomien übertragen. Entsprechend sollte auch hier der Risikofaktor Rauchen im Vorfeld Berücksichtigung finden und die Patienten sollten gerade bei elektiven Eingriffen darüber aufgeklärt werden.

\section{Martina Wendt}

Chirurgische Klinik und Poliklinik der Universität Rostock, Abt. für Unfall-, Handund Wiederherstellungschirurgie martina.wendt@med.uni-rostock.de 